\title{
Sector spanning agrifood process transparency with Direct Computer Mapping
}

\author{
Mónika Varga ${ }^{1}$, Sándor Balogh ${ }^{2}$, Béla Csukás ${ }^{3}$
}

\section{N F O}

Received 19 Oct. 2010

Accepted 24 Nov. 2010

Available on-line 30 Nov. 2010

Responsible Editor: K. Rajkai

Keywords:

agrifood, sector spanning traceability, transparency, interoperability, Direct

Computer Mapping

\begin{abstract}
A B S T R A C T
Agrifood processes are built from multiscale, time-varied networks that span many sectors from cultivation, through animal breeding, food industry and trade to the consumers. The sector spanning traceability has not yet been solved, because neither the "one-step backward, one-step forward" passing of IDs, nor the large sophisticated databases give a feasible solution. In our approach, the transparency of process networks is based on the generic description of dynamic mass balances. The solution of this, apparently more difficult task, makes possible the unified acquisition of the data from the different ERP systems, and the scalable storage of these simplified process models. In addition, various task specific intensive parameters (e.g. concentrations, prices, etc.) can also be carried with the mass flows. With the knowledge of these structured models, the planned Agrifood Interoperability Centers can serve tracing and tracking results for the actors and for the public authorities. Our methodology is based on the Direct Computer Mapping of process models. The software is implemented in open source code GNUProlog and C++ languages. In the first, preliminary phase we have studied a couple of consciously different realistic actors, as well as an example for the sector spanning chain, combined from these realistic elements.
\end{abstract}

\section{State-of-art: traceability of agrifood process networks}

Traceability has been interpreted in several ways in the literature and in regulations. In common sense we can say that it covers the ability to identify and follow the units of raw materials or products through the process network, unambiguously. The definition of the TRU (Traceability Resource Unit) has been determined by Kim. According to this formulation, TRU is a clearly defined unit, that is uniquely identifiable and traceable (Kim et al., 1995).

Traceability system originally means mainly record keeping procedures, which show the path of the TRUs, through a part of the production processes. We can classify the existing information management systems into the following groups:

- Internal (within actors): follows data about raw materials and processes within the business, connected to the final product in each stage of production, processing or distribution, separately. Several studies are available in this topic (Bertolini et al., 2006; Dupuy et al., 2005), and there are also sectorial investigations about the applications of IT systems (Füzesi and Herdon, 2010).

- External (among actors): means traceability between various authors in the chain (Jansen-Vullers et al., 2003).

\footnotetext{
${ }^{1}$ Mónika Varga

Kaposvár University, 40 Guba S Kaposvár, 7400, Hungary

varga.monika@ke.hu

2 Sándor Balogh

Kaposvár University, 40 Guba S Kaposvár, 7400, Hungary

balogh.sandor@ke.hu

3 Béla Csukás

Kaposvár University, 40 Guba S Kaposvár, 7400, Hungary

csukas.bela@ke.hu
}

ISSN 2061-862X http://www.magisz.org/journal

Mónika Varga, Sándor Balogh, Béla Csukás:Sector spanning agrifood process transparency with Direct Computer Mapping 
- Whole chain system: aims spanning the sectors by means of separated, upper level, external databases (Opara, 2003; Calder and Marr, 1998).

In case of internal tracing and tracking systems, we can detect a duality. In one hand, large enterprises use effective ERP integrated systems, but SMEs or small private businesses are usually not able to operate these systems, on the other. There is another duality in the field of sector spanning traceability. Two extremely different approaches have been formed. First is the solution with standardized IDs, according to the EU regulations, namely to the one-step-up, one-step-down principle. But in this way, we lose the most important thing, the transparency of the whole network. The other approach plans huge central databases, where all data of the various actors are stored. Because of the widespread character of the agrifood network, of the heterogeneity of the stakeholders, and of the large amount of data, it seems unreal. We can state, that the good solution, the middle course does still not exist.

In the last decades, forced by a growing, conscious consumer society, an increasing demand appeared for the healthy and safe food products. It is easy to understand that transparency can be guaranteed only by the forward and backward traceability of the whole system in a sector spanning way, from the cultivation, to the trading sector. Schiefer emphasizes, that because of food chain complexity, enterprises cannot solve the whole chain problem alone, but it requires concerted action (Schiefer, 2008).

Motivated by the various food scandals, an assertive demand has been appeared to handle, to enable and to share the huge amount of collected information, through the whole chain, already in the first years of the 21 st century.

Numerous projects have been carried out in this field since then, but we can say that the problem is almost the same, as twenty years ago. The various food scandals resulted several methods and solutions. In a recent paper Wolfert et al. describe explicitly, that a really workable sector spanning system has not yet been developed (Wolfert et al., 2010). A more comprehensive state-of-art review can be found in our former paper (Varga and Csukás, 2010).

\section{Outlines of a possible solution}

In general, traceability in the agrifood networks means the ability to follow a food, feed, foodproducing animal or substance through the whole chain of production, processing and distribution, from the field to the table of the consumer. According to our understanding, from a process modeling point of views, it means the tracing and tracking of stoichiometric balance processes through the whole process chain.

In our paper we outline the characteristics of a possible solution, based on the Direct Computer Mapping (DCM). Our paradox concept is that we have to solve an apparently more difficult task. This task is the tracing and tracking of dynamic mass balances. It seems difficult at first, however it provides us the transparency of the whole network. The dynamic mass balance of the input and output TRUs of an actor means also the necessary and sufficient information to describe the structure. With the simulation of the actual data set, we can fix the respective actual state. In this context, further simulation means the extension of the database stepwise, in line with the data acquisition. The acquisition of the dynamic mass balances can be solved with unified principles from the ERP systems. Of course, for the smaller actors we have to give appropriate software to this activity. In this way the various task specific intensive parameters (e.g. prices, concentrations) can also be carried together with the mass flows. This solution supports the tracing and tracking of suddenly appearing components by effective searching algorithms.

The above outlined characteristics claims for an IT solution, that supports the model generation from unified building elements, helps the scalable storage of the model files in databases, makes possible the case specific extension of the models, and supports the development of effective multiscale tracing and tracking algorithms. 


\subsection{Principles of the method}

The principle of Direct Computer Mapping has been elaborated many years ago (Csukás and Perez Uriza, 1995; Csukás, 1998; Csukás and Balogh, 1998; Csukás et al., 2005), and since then, it has several practical applications in those fields, where difficult problems required a non-conventional, but flexible solution.

The basic idea behind the principle can be summarized by the followings. In the usual way of problem solving, we build a mathematical construct from simple equations or rules, via abstractions. Next, we have to decompose it, and solve with a numerical method. The DCM approach simplify this way. Its basic concept is, that "let know the computer about building elements of the real world". In this way, according to Figure-1., we map the problem directly from elementary building blocks into the executable program.

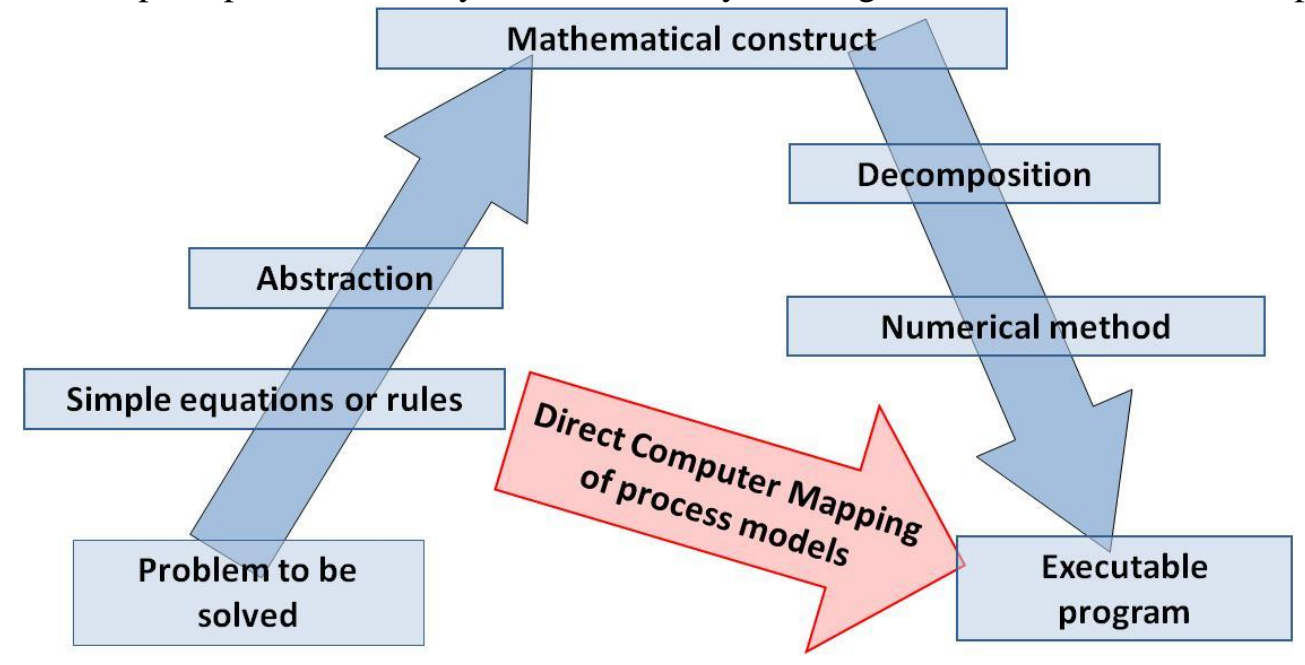

Figure 1. Principle of Direct Computer Mapping (Csukás, 1998)

The determination of these model elements comes from the natural structure of the process models. There are states (measures or signs) and transitions (transportations, transformations or rules) behind all of the processes. These elementary building blocks communicate with each other, and with their environment. In the software implementation these natural elements and their connections can be associated with brief program code behind them. It means, that the discrete or continuous and quantitative or qualitative functioning of the state and transition elements can be described by typical classes of expert defined brief programs, executed by the general kernel. The general kernel contains those functionalities that are indispensable for the simulation (e.g. time handling, etc.).

According to the experiences of the former applications, the various processes can be generated from the same, unified building blocks, and it gives the general applicability for the method.

There are some exciting and useful principles behind the Direct Computer Mapping. The conservational processes can carry informational sub-processes. The difference between them is that informational processes consume less resources, and effects more impact on the complementary part, than vice versa. In the practical applications, according to this principle, the qualitative knowledge can be carried as intensive parameter with the mass flows, quantitatively.

The other exciting outcome is the principle of cooperative processes. It means, that cooperation is organized by the mutual (consensus) consideration of the objectives between the functionally connected neighbors. The practical application of this principle in our case is, that stakeholders of the system serve and request traceability information according to the cooperative scheme. 


\subsection{Software implementation and interfaces}

The software implementation of DCM applies declarative logical GNU-Prolog and C++ languages. For graphical user and expert interfaces, we use an extended Graphviz (Varga, 2009), temporarily, while there is an ongoing development for a new graphical user interface, based on Qt, which is a cross-platform application and UI framework. (Internet1).

Right now, the investigated process structures are described by an extended Graphviz interface. We describe graphically the elements of the processes according to the principles of DCM. In line with it, Graphviz map the structure automatically into a simple .dot format. We extend this .dot format with GNUProlog declarations, which makes possible the description of the characteristics according to the investigated process (e.g. the actual measures, the initial concentrations, etc.). The kernel program generates the user and expert modules automatically from this file, next executes the dynamic simulation, and write the results into a simple .csv file.

\section{Example applications of the developed method}

To test the applicability of the method and the effectiveness of the developed tracing and tracking algorithms, we tried to choose consciously different stakeholders, with quite different activities. In Table1. we summarize the main characteristics of the studied examples.

Table 1. The investigated examples

\begin{tabular}{|c|l|l|l|}
\hline Investigated system & \multicolumn{1}{|c|}{ Case study 1. } & \multicolumn{1}{|c|}{ Case study 2. } & \multicolumn{1}{c|}{ Case study 3. } \\
\hline model farm)
\end{tabular}


In every case the most important issue was to outline the structure on the basis of the previous year's data. In this way, we fixed the initial state. The recent task is the real-time, continuous data acquisition, depending on the characteristics of the investigated system.

\subsection{Arable farming}

First analyzed example was a real model farm, with arable farming activity. The state elements were the parcels, as well as the input and output storages. The transition elements were the various agricultural and commercial processes with mass flows. We can use the mandatory data registers as data sources. The frequency of data acquisition is generally determined by the characteristics of the investigated processes. In this case, the 1-2 weekly data acquisition seems to be a good practice. Collected data are the followings:

- data about the cultivated parcels;

- data about the utilized raw materials, in TRU units (seeds, fertilizers, pesticides, etc.);

- data about the products, in TRU units;

- seller and buyer information.

In the recent phase of the development, we are working on the automation of the data acquisition with unified principles, on the basis of the obligatory data registers. The basic principle is, that the necessary and sufficient data are the mass balances of the TRUs.

All of the examples have their special difficulties. In case of arable farming, one of the biggest problems is the permanent, time to time changing of the parcel structure. For example, it makes difficult the tracing of the various soil components, which come with the fertilizers, etc.

These problems mainly originate from the not really well-considered record system. For example, the administrative name and measure (by the satellite) of the parcels appear only after the physical cultivation, next year. Another problem is that the measured data are often incorrect, although, it is the basis of the agricultural subventions. Figure-2. illustrates a small part of the investigated crop farming, regarding the parcel No.22.

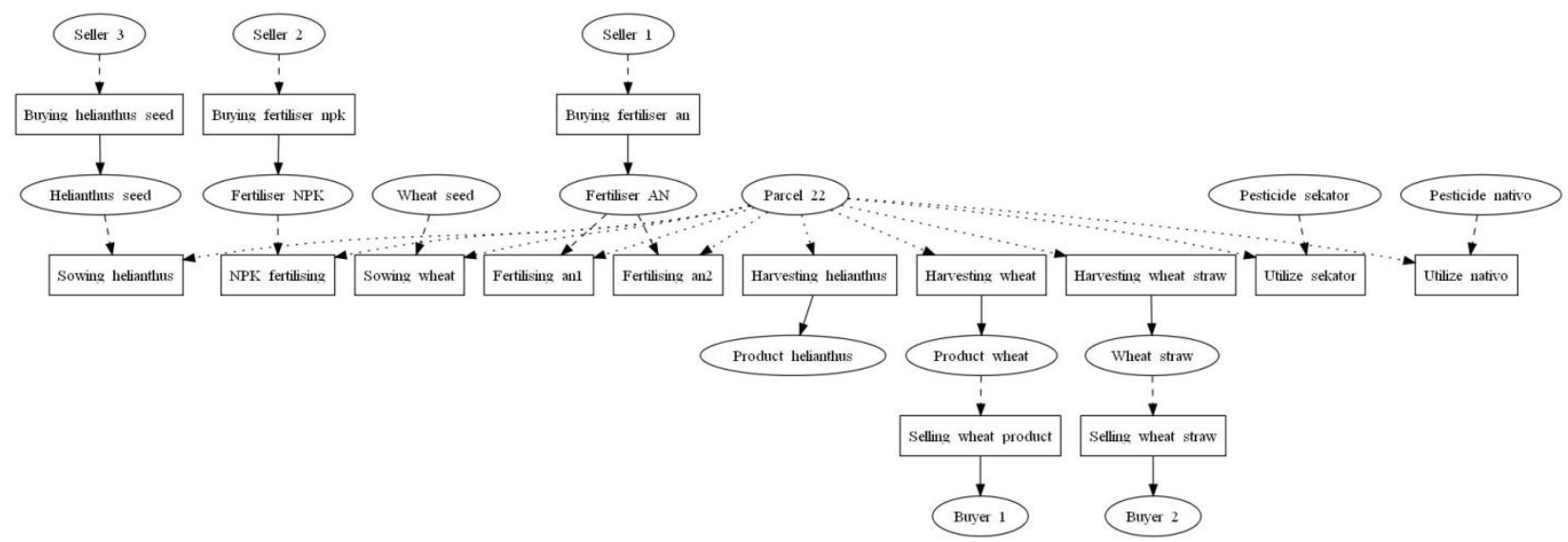

Figure 2. A small part of the arable farming example (parcel No.22)

On the basis of the whole structure (altogether 47 parcels), the kernel program of the simulator generates automatically the necessary files for the dynamic simulation.

Simulation results, based on the previous crop years' data, provide a good basis for the initial state. Recently, we extend the database continuously, as well as we develop the data acquisition software and the quantitative tracing and tracking algorithms. 
In the following diagrams we illustrated some results of the dynamic simulation, on the basis of the previous two crop years. In Figure 3. we can see a discrete event, the changing of the parcel name. In this example, there was no change in the measure of the area. In Figure 4., we can follow dynamically the utilization of the seed and the harvesting of the product. Figure 5. shows the consumption of the various fertilizers.

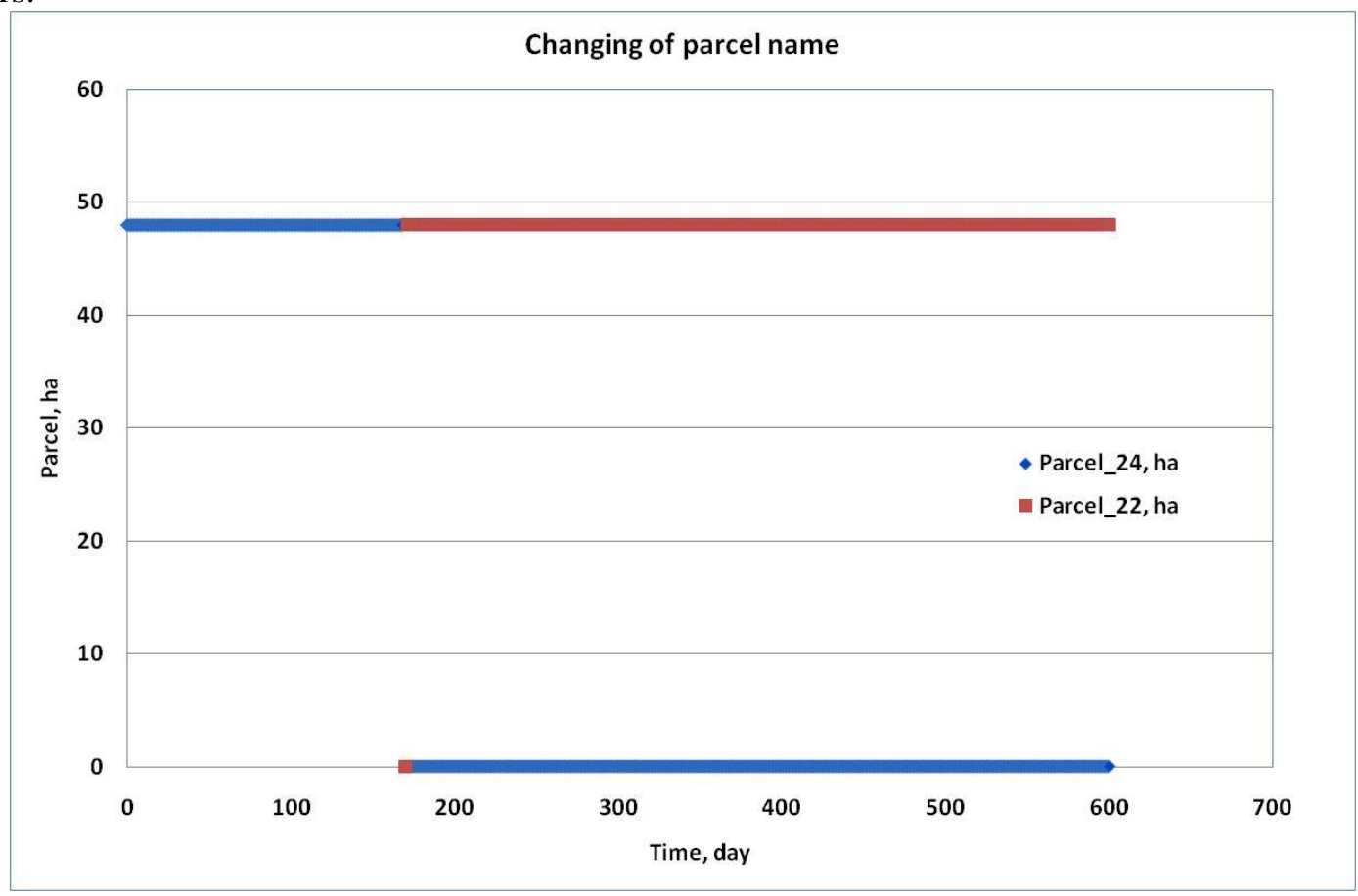

Figure 3. Dynamic simulation of a discrete event

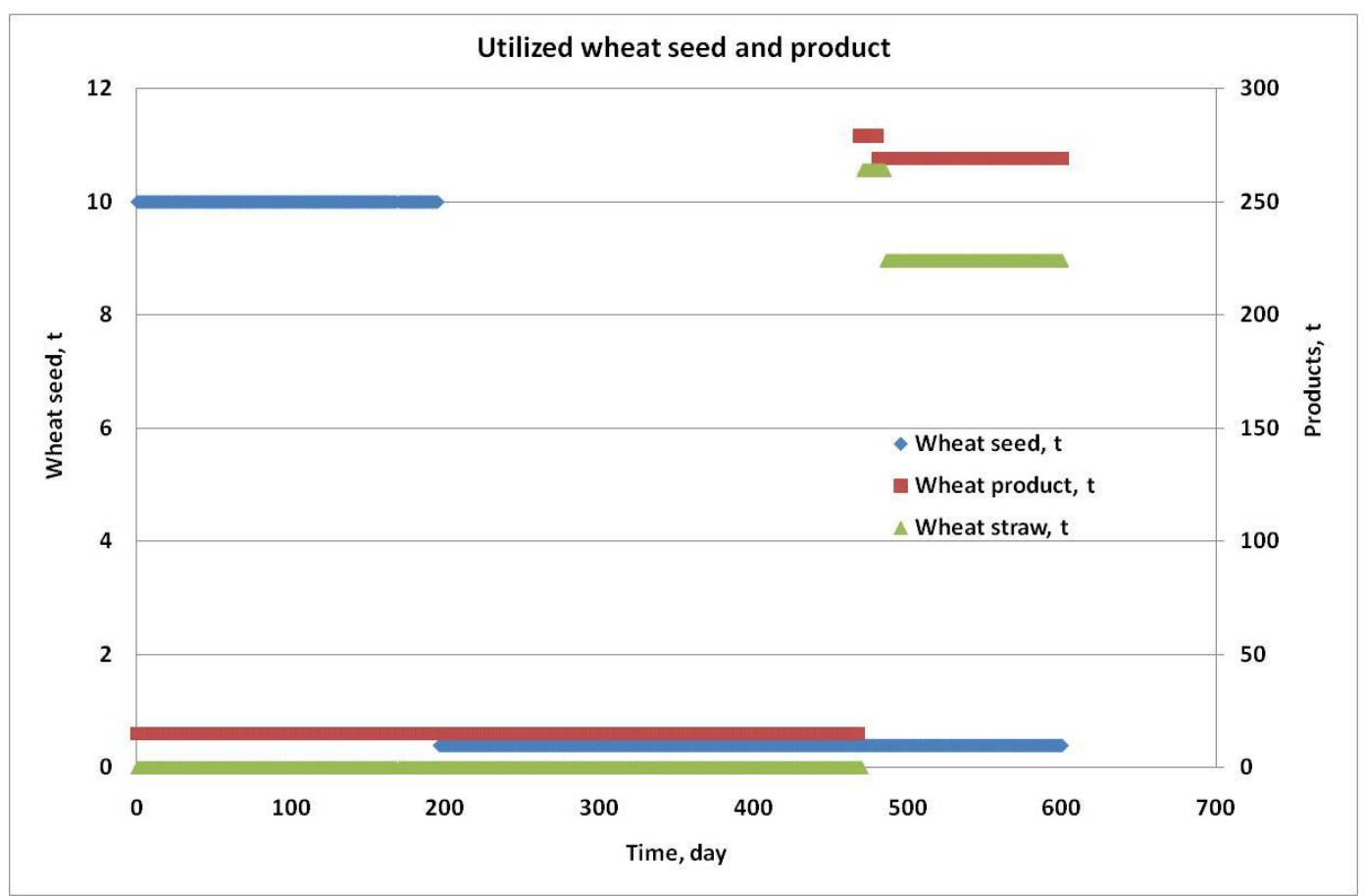

Figure 4. Dynamic simulation of some raw material and products 


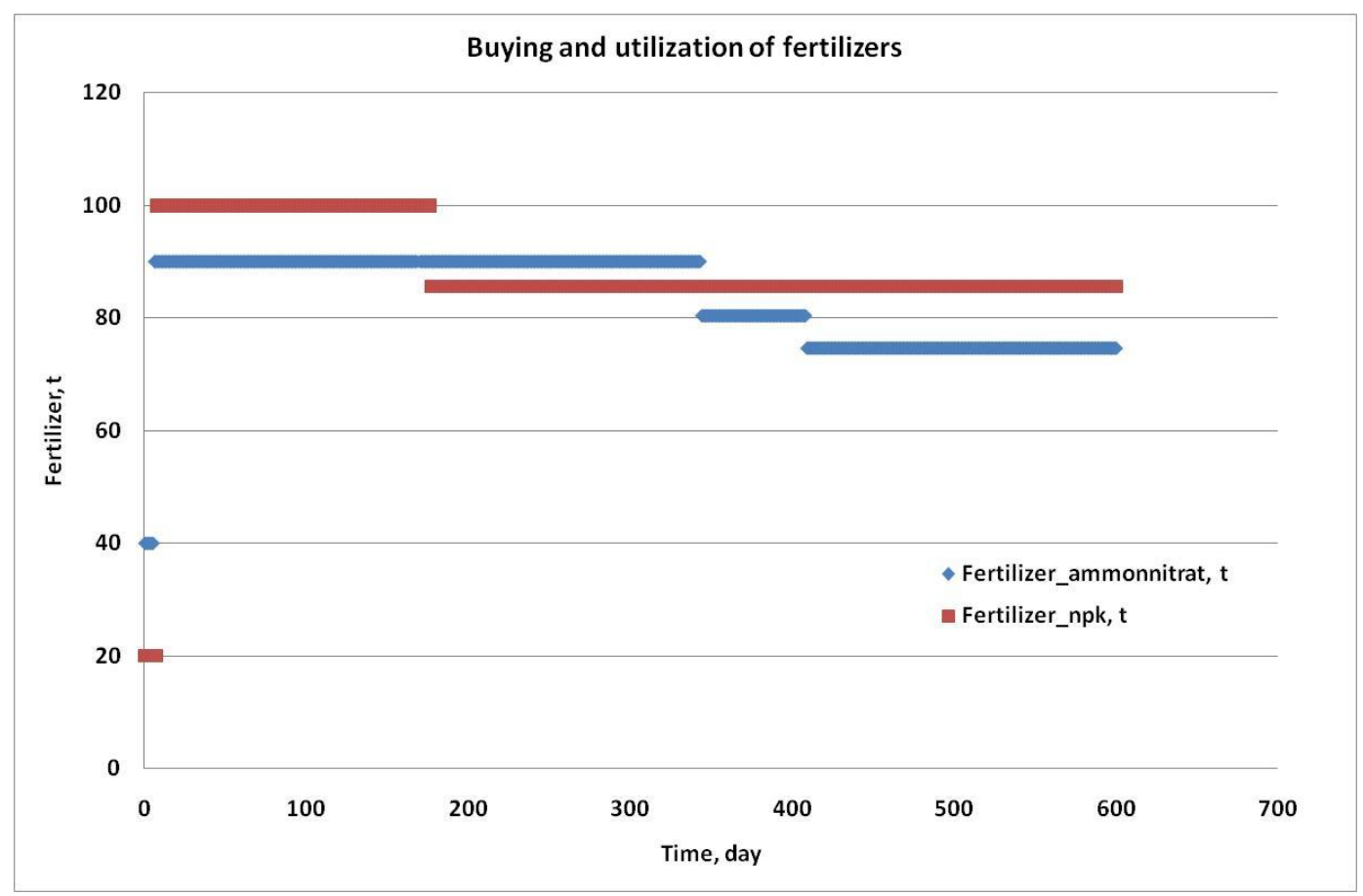

Figure 5. Simulated amount of various fertilizer pools

Using the developed Prolog algorithms, we can search for the origin of a given component, or we can track the way of a given component, until all of the possible outputs. In this application the program lists the possible routes into a .csv format.

\subsection{Game management}

A quite different example was a real company, dealing with game management in almost 1.5 thousand hectares. According to the valid regulations, they register the animals individually. Similarly to the above example, we determined the process elements (see in Table 1.), built the structure and, with the actual data, we simulated the previous year, 'a posteriori'. In the same way as in the arable farming example, we can follow the dynamically changing data of the involved components with the forward simulation.

In this case, the age and season depending feeding strategies made the problem solving more difficult. To eliminate this problem, we utilized the advantages of the above mentioned theoretical principle, namely the specific interpretation of the conservation based informational processes. Accordingly, we determined the various feeding strategies as signs.

Another exciting characteristic of the applied method is the ability for the backward simulation. Figure 6. illustrates the results of the inverse simulation. As the dynamic simulation provides basis for the tracking, inverse simulation supports the tracing functions. In this case, starting from a fixed final state, the kernel executes the backward simulation, with the knowledge of the equations, used for the simulation. In this Figure we can see the forward and backward simulated changes of weight of a given individual. 


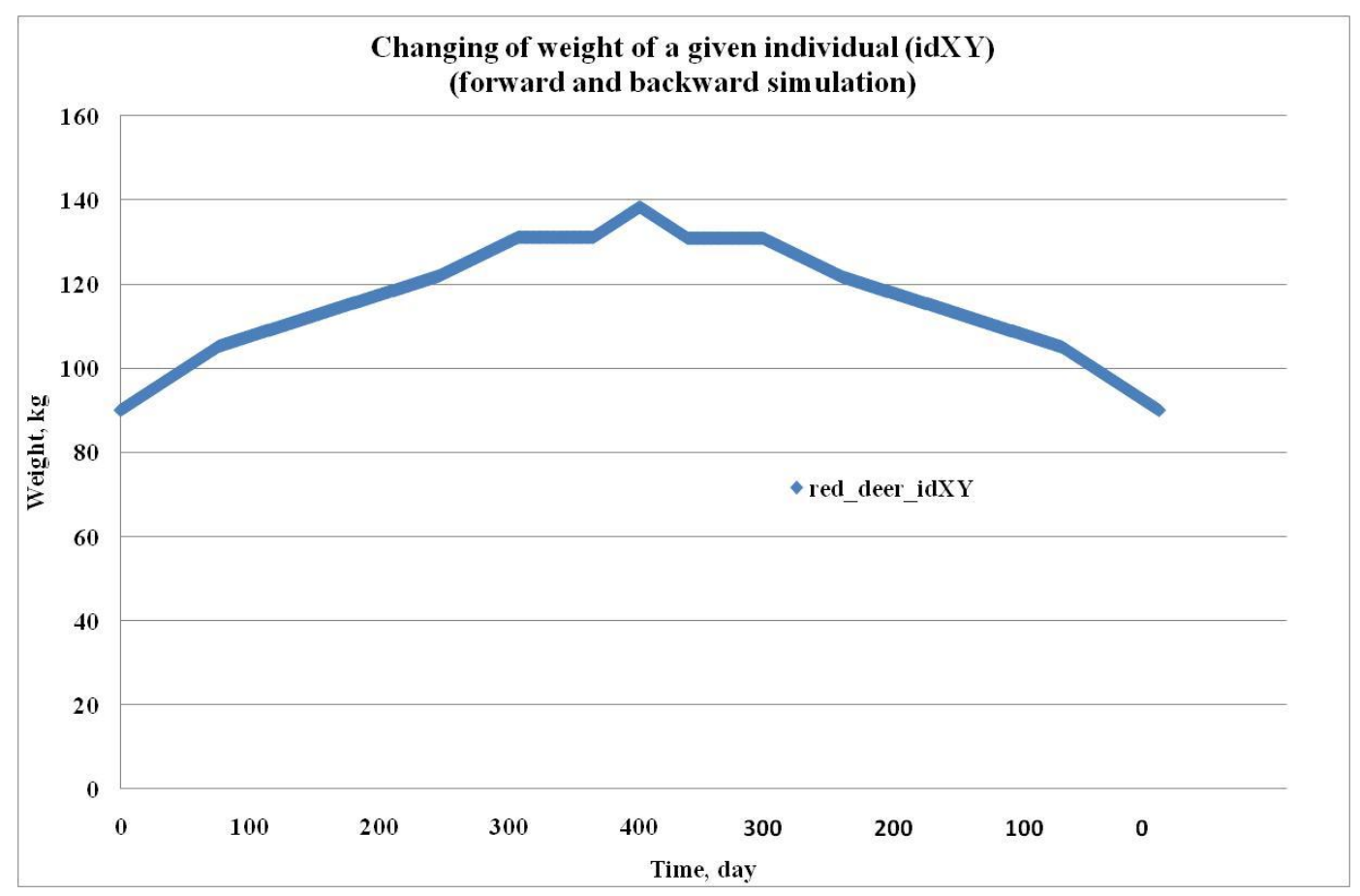

Figure 6. Forward and backward simulation of the weight of a given individual

\subsection{Slaughterhouse}

Third example was a slaughterhouse, modeled similarly to the other examples. According to the experiences, there are special difficulties to be solved, but the description of the structure and functionalities can be done in the same way.

To make the data acquisition easier and to eliminate the additional data needs, we tried to lean on the mandatory data registers in this case, too. Of course, we adjust data acquisition to the frequency of the slaughterhouse processes. It means hourly data collection.

Special difficulty was the identification of packages, produced from different carcasses. We trace the package ID back to the flock ID. In this way, we resolved the problem, and it makes possible to reach the supplier.

\section{Current research}

Recently, we try to combine the previous examples and to study, how the heterogeneous actors can be joint in various searching algorithms.

Figure 7. illustrates the connection of the above described three actual examples. It is a quite fictitious and arbitrary process, of course, but it gives a good basis for the forthcoming development. The connecting elements are the commercial processes, obviously.

According to our basic concept, the way of sector spanning tracing or tracking can be explained as follows. For example, let's see the case of a suddenly appearing unknown component. Firstly, we are search for the possible routes of this component, only in the inputs and outputs of the involved stakeholders (higher level, sector spanning structure, signed with red circles in Figure 7.). 


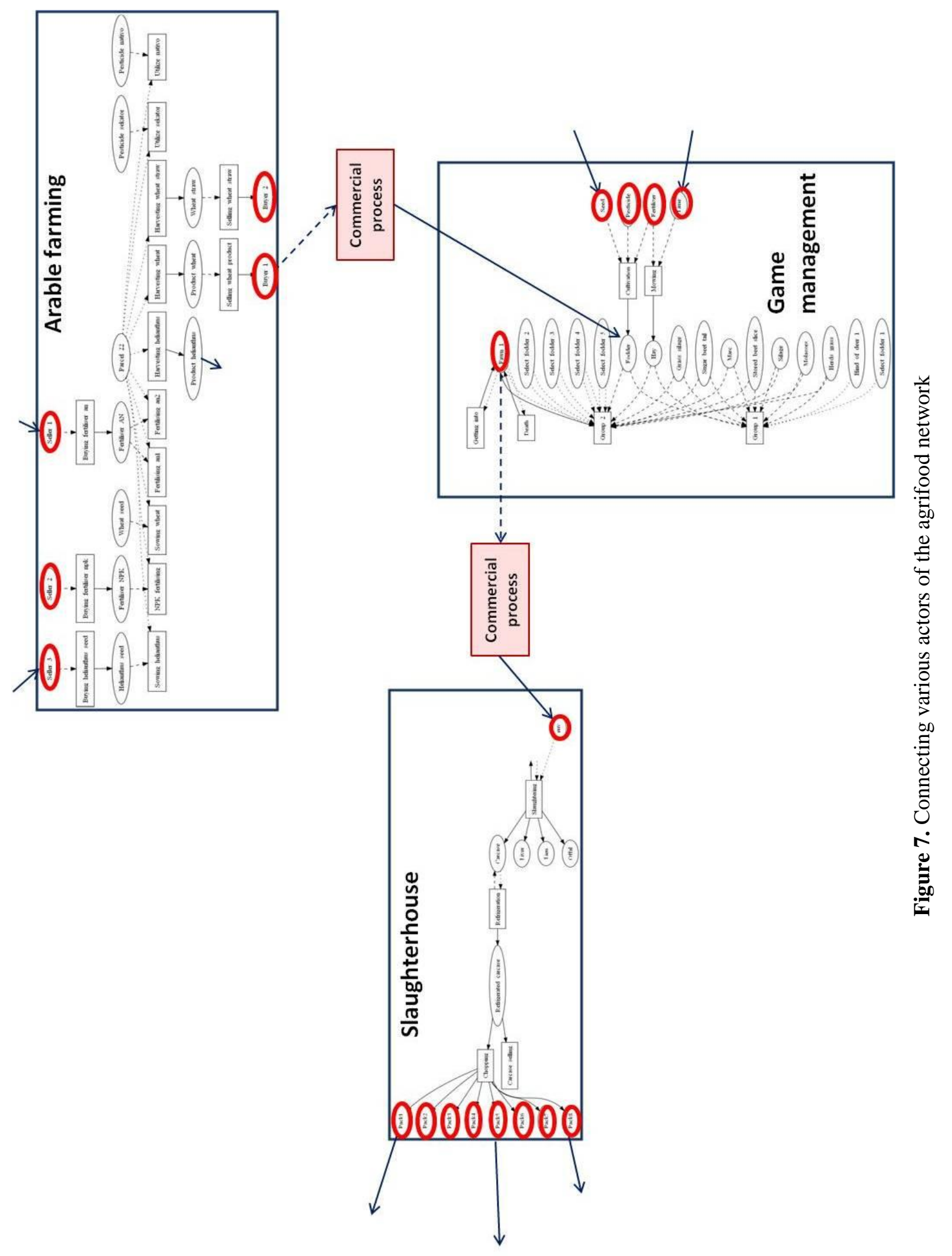


Next, if there is a path until a given input or output, we trace into the macro level model of the actor, which practically means the stored bone structures of its processes, described by the dynamic mass balances and stoichiometries. These two stages make more effective the search, especially in case of huge networks. Another advantage is that with linking of the new component to the mass balances, we will be able to follow such new components that are not involved into the model before. In this way, the Center will be able to suggest measurement points and/or can makes measurements.

Our final goal is an example region, with a sector spanning traceability system. Of course, it needs further improvements, with special regards to the data acquisition tools and the tracing and tracking algorithms. The step-by-step extension of this example network claims for the clear determination of the system contour, and for the absolute indication of the not involved sources.

\section{Conclusion}

A daily problem for the actors, that the frequently changing mandatory data demands are overlapping and sometimes confusing. Various authorities ask for data from various aspects, and in spite of it, data set is still not complete. Another serious problem is, that the required data does not satisfy the observability and controllability of the processes.

Probably, a great byproduct of our work would be if the authorities would realize that mandatory data demand needs better considered coordination and rationalization. Another advantage is that the developed methods will support not only the solution of the traceability, but also other important tasks (e.g. value chain analysis, observation of hidden resources). We can state, that the method makes possible the unified management of the completely different processes. According to our experiences, effective tracing and tracking algorithms can be developed with the applied declarative, logical programming language. The various intensive parameters can be carried by the dynamic mass flows. For example, we are able to carry with the mass flows various components that appear suddenly. The acquired process data can be stored in the dedicated databases of an Agrifood Interoperability Center. On the basis of the developed methods, the role of planned Agrifood Interoperability Center would be the data serving for actors and authorities, based on the real data and/or on the simulated case studies. The tasks to be solved are e.g. the forward and backward tracing investigations, the determination of hidden resources, value chain analysis, or waste detection.

Considering these tasks, the necessary data exchange can be summarized in the following. The Center would need the input and output mass flows in TRU units, the stored amount of TRUs, the calculated or estimated stoichiometries between the TRUs, and possibly, additional knowledge that supports special investigations. The Center serves for the actors tracing and tracking information, and in case of harmful components, suggest measurement points or realize the measurements itself.

An exciting outlook for the future is that the agrifood process interoperability could be a good example for the future economic paradigm. Agriculture utilizes the single outer resource of Globe, the solar energy. Food serves the bare necessities of mankind. Interoperability supports local supply, which will be more and more important from strategically and economical points of views in the future. That is why the dedicated Agrifood Interoperability Centers must be out of the various lobbies and individual interests.

\section{Acknowledgements}

This study was funded by Baross project (REG_DD_KFI_09, FKM_KE09).

\section{References}

Bertolini, M., M. Bevilacqua, R. Massini. 2006. FMECA approach to product traceability in the food industry. Food Control, 17: 137-145. 
Calder, R., P. Marr. 1998. A beef producer initiative in traceability: Scottish Boarders TAG. Supply Chain Mtg., 3(3): 123-126.

Csukás B, S. Balogh, Gy. Bánkuti. 2005. Generic Bi-layered Net Model - General Software for Simulation of Hybrid Processes. 2nd IFIP Conference of TC12 WG. May 12. 1995. In: Artificial Intelligence Applications and Innovations II. (Eds.: Daoliang Li, ÍBaoji Wang). 701-710.

Csukás B., S. Balogh. 1998. Combining Genetic Programming with Generic Simulation Models in Evolutionary Synthesis, Computers in Industry 36: 181-197.

Csukás B., S. Perez Uriza. 1995. Discrete Modelling by Direct Mapping of the Conservational Processes, Hung. J. Ind. Chem., 23(4): 277-287.

Csukás, B. 1998. Simulation by Direct Mapping of the Structural Models onto Executable Programs. AIChE Annual Meeting, Miami, Paper 239/9.

Dupuy, C., V. Botta-Genoulaz, A. Guinet. 2005. Batch dispersion model to optimize traceability in food industry. Journal of Food Engineering, 70: 333-339.

Füzesi, I., M. Herdon. 2010. IT applications in the quality management of Hungarian meat production chains. Agricultural Informatics, 1: 19-29.

Jansen-Vullers, M. H., C.A. van Dorp, A. J. M. Beulens. 2003. Managing traceability information in manufacture. International Journal of Information Management, 23: 395-413.

Kim, H. M., M. S. Fox, M. Gruniger. 1995. An Ontology of Quality for Enterprise Modeling. IEEE Proceedings of WET-ICE, 105-116. Los Albamitos, CA, USA.

Opara, L. U. 2003. Traceability in agriculture and food supply chain: A review of basic concepts, technological implications, and future prospects. Food, Agriculture \& Environment, 1(1): 101-106.

Schiefer, G. 2008. Tracing and Tracking - A Challenge for System Organization and IT. Journal of Information Technology in Agriculture, 3: 19-25.

Varga M. 2009. Economic Optimization of Sustainable Complex Processes Under Uncertain Cost Parameters. Regional and Business Studies, 1(2): 57-64.

Varga M., B. Csukás. 2010. On the Way toward the Sector Spanning Agrifood Process Traceability. Agricultural Informatics 1(1): 8-18.

Wolfert, J., C. N. Verdouw, C. M. Verloop, A. J. M. Beulens. 2010. Organizing information integration in agri-food - A method based on a service-oriented architecture and living lab approach. Computers and Electronics in Agriculture, 70(2): 389-405.

Internet1: http://qt.nokia.com 